For the moment more important is the issue of refusal by relatives. The style and professionalism of the approach to relatives are paramount. Tired, half hearted, untrained, uninformed, and embarrassed requests fail. Referral to transplant coordinators or other identified trained staff within the hospital helps both the staff and the relatives who are faced with difficult decisions at times of great personal stress. Such referrals reduce the number of organs refused, and Gore and her colleagues have suggested that they should be "required." Many relatives, having refused donation, regret the decision later. This is doubly tragic and could be avoided by a sensitive and professional approach.

Having attempted to maximise the total available number of donors, how best are we to use the organs? Firstly, we must make sure that these are usable. The quality of donor organs reflects the standards and quality of donor care. This is particularly important for heart, lung, and liver grafts, which need to function fully immediately after transplantation. Not all of the donors identified in Gore's paper would have been suitable on grounds of size or age, and many would have had unsuitable organs as a result of trauma, resuscitation efforts, and general deterioration. The patient with brain stem death has loss of vasomotor tone, temperature control, and hormonal imbalances, and care is not easy; an unknown number of organs become unsuitable for transplantation because of inadequate care at the donor hospital. Extra facilities and education would reduce this number-again, further audit may identify whether this number is substantial. Fortunately, multiorgan donation from a single donor is on the increase, but within Britain the proportion of donors offered for organs other than kidneys varies from region to region. Overall, the figure quoted by Gore et al-60\%-is encouraging, but it could be improved. Nevertheless, within the total number of potential donors identified not all would be suitable for multiorgan donation as stricter criteria are necessary for heart, heart-lung, and liver transplantation. Limitations on ischaemic time vary for the different organsup to four hours for heart and lungs and eight to 12 hours for livers compared with more than 24 hours for kidneys.

Other sources of difficulty are the inadequacies and inequities in the organisation of the distribution and use of donor organs within Britain. The functions of transplant coordinators need to be more clearly identified and the career structure improved. The United Kingdom Transplant Service is not a statutory body, and though it is expected to monitor and guide the use and supply of donor organs, it has neither the power nor the facilities to run a comprehensive national system. Much is being achieved, however, with the recent introduction of registration of transplants and an increase in staff so that the service is better able to provide 24 hour cover.

Clearly, however, even with all these potential improvements in the supply of organs many patients will not receive transplants and will remain chronically sick or die. The best use must be made of the available organs, and that implies careful and accurate audit. Such assessments may highlight conflict between the needs of an individual patient and the overall best use of the organs available. For clinicians faced with sick patients this is a dilemma that may worsen as the gap widens between the demand for transplantation and the supply of organ donors. Transplanting moribund patients and those at high risk is not the best way of using donor organs except in particular circumstances (for instance, sometimes in liver transplantation). The objective should be to have most donor organs functioning five to 10 years after transplantation. Deterioration in the condition of patients waiting long periods for donor organs is another factor that may affect eventual outcome both in terms of additional risks to the patient and extra resources needed to nurse sick patients in intensive care after transplantation.

Transplantation of human organs is and will continue to be a restricted activity, and transplantation services need to be planned and monitored to ensure the greatest long term benefit to the maximum number of patients. Unplanned activity for apparent local benefits may dilute both experience and skill. The ultimate goal must, however, be to find an alternative to human allograft transplantation. Research and interest are now being focused more intensively on transplantation from animals with the prospect of organs of reliable quality being available in bulk. Only then will transplantation be available "when you want it, where you want it, and by whom you want it." Until that time, every organ is precious and should be used efficiently and wisely.

Consultant Cardiothoracic Surgeon,

J WALLWORK

Papworth Hospital,

Cambridge CB3 8RE

1 Buxton M, Acheson RM, Caine N, Gibson S, O'Brien B. Costs and henefits of the heart transplant programmes at Harefield and Papaworth Hospitals. London: HMSO, 1985.

Gore S, Hinds CJ, Rutherford AJ. Organ donation from intensive care units in England: first report. Br.Med f 1989;299:1193-7.

Roels L, Vanrenterghem Y, Ware M, Gruwez J, Michielsen P. Effect of a presumed consent law on organ retrieval in Belgium. Transplant Proc (in press).

\title{
Mental health for all?
}

\section{We should identify discrete targets}

The World Health Organisation's Alma Ata declaration invited the nations of the world to attain "health for all by the year 2000." Though there may be doubts about its realism, this objective has certainly stimulated debate, particularly about public health strategies. The many issues that the slogan raises are especially difficult in psychiatry.

The first question is just what is meant by "mental health." Psychiatrists have agonised for decades over the problem of defining health and disease, although colleagues in other disciplines appear to have little interest in the formal delineation of the concept of disease. Yet within psychiatry no satisfactory formulation has been reached-possibly because the concept of disease sheds little direct light on the "normal" or "abnormal" processes that concern us. The essential task for both the clinical psychiatrist and the researcher is to understand how social, somatic, and psychological processes interact and how they sometimes lead to feelings of distress. If and when such a systematic understanding is reached a secondary distinction may then be drawn identifying those types of distress that are appropriate for the medical disciplines, broadly defined, and those that are not. Such a division will inevitably be influenced by a host of moral assumptions, historical determinants, and social constraints. At the end the designation of disease will act as a pointer to 
action-but the debate will then have moved away from fundamental scientific issues concerning the genesis of mental states.

This view, which is close to what is often termed the social concept of disease, brings its own difficulties, and until these are resolved no one is in a strong position to pronounce on the definition of mental health. The approach nevertheless has certain advantages, including its recognition of the arbitrary way in which conditions are designated as requiring to be prevented or treated. There is a large measure of agreement about these, as reflected in standard taxonomies such as the International Classification of Diseases. Yet even that distinguished publication includes some rule of thumb decisions. Few would doubt that patients with schizophrenia or Alzheimer's disease fall outside the rubric of the healthy, but with the common neuroses or personality disorders we are dealing with extremes of distributions rather than categorical distinctions. In such cases - as with, say, hypertension - the dividing line must be based on informed judgment rather than logic. A solution of this kind may be untidy, yet it may serve us best for practical purposes.

Assuming, then, that we have provisionally identified the condition we wish to tackle and are fortified by some extensive epidemiological knowledge of psychiatric disorder, the next problem is to devise and apply preventive strategies. Again, that is not easy. With some conditions, such as alcohol abuse, plans for a detailed public health approach have already been advanced - for example, by the royal colleges.' The government (and perhaps society in general) seems reluctant to act, but a strategy can be and has been mapped out. Yet what are we to make of a recent publication on the prevention of mental disorder that stipulates "peace, social justice, decent housing, education, and employment" as essential components of a preventive programme? ${ }^{2}$ No one is against such objectives, but perhaps it would be more rewarding - at first, at least - to concentrate on more specific efforts ranging from better antenatal care and improved physical and psychological health of schoolchildren to counselling for the bereaved and community management of the elderly. It would be better to succeed piecemeal than to fail comprehensively.

NORMAN KREITMAN

Director and Honorary Consultant,

Medical Research Council Unit for Epidemiological Studies in Psychiatry, Royal Edinburgh Hospital,

Edinburgh EH10 5JH

Anonymous. Royal colleges unite to fight alcohol misuse. L.ancet 1987;ii:1162.

2 Anonymous. Mental health. Health for All by the Year 2000 News Spring 1989 (no 8): 1 (Faculty of Community Medicine, Royal Colleges of Physicians.)

\section{Audit in general practice}

\section{Two track programme needed}

The Oxford English Dictionary defines audit as "a searching examination, $c f$ Day of Judgement." The medical profession seems to have found the concept of "audit" one of the better bits of the white paper curate's egg, but there may be substantial differences of intent among general practitioners, family practitioner committees, managers, and the Treasury. The government will hope to find out what it is getting for its money; general practitioners will want to close the gap between what they think they are doing and what actually gets done; and managers will want to use audit to drag the tail of the caterpillar toward the head. A programme with three different goals is fraught with problems.

Audit is defined in the draft departmental circular $\mathrm{HC}(\mathrm{FP})(89)$ - which is now available for consultation-as "the systematic critical analysis of quality," but none of the words are defined. General practice is a complex mix of activities (prevention, the care of patients with acute and chronic illness, and terminal care) that aims not only to be comprehensive (all illnesses, all population groups) and continuing (all phases) but both in formulating problems and planning treatment to take into account physical, social, and psychological factors.

A "systematic" examination will have to take a balanced view of all these activities. A "critical analysis" suggests that there are valid measures available to be applied to the care given, but very few of the measures of process that make up much conventional audit have been or even could be validated by measures of outcome.

We all have strongly held ideas about what constitutes "good" care in general practice, but whether such care makes much difference to the outcome perceived by the patient is seldom proved one way or the other. "Quality" has three different components (which might well be put in a different rank order by each of the three parties referred to above, let alone by the "consumer"): effectiveness, efficiency, and acceptability. By definition, if care is not effective (that is, it improves the outcome in terms of measured health status) there is no point in seeing if it was efficient (effectiveness gained with minimal use of resources). If the whole thrust of the white paper is toward the citizen consumer then acceptability becomes paramount, even though there will be times when maximum acceptability - in terms of the patients getting what they want - may be neither effective nor efficient.

The draft circular pays far more attention to structure and administration than to these fundamental questions as to the philosophical and scientific basis of audit. Its statement that data from the family practitioner committees and district and regional health authorities must be available to the Medical Audit Advisory Group suggests that it sees data from these sources as identifying "outliers" who could then be submitted to (or encouraged to undertake) audit. Homogenisation of services is no doubt attractive to managers, but is it realistic, and do these data give useful information about the day to day activities of the practitioner such as ordinary consultations?

I believe that what is needed is a "two track" programme of internal and external audits. General practitioners should be helped to set up and run practice information systems that will allow them to answer the question, "Is what I think I am doing what really gets done, and if not, why not?" The costs need not be very high. Educational initiatives should be aimed at helping general practitioners to formulate such questions across the whole range of their activities and to answer them reasonably objectively as a basis for improving their practices where needed. At this level it is less important that the measures used should have been validated against outcomes because the first question must be, "Have I got a clinical policy, and can I achieve it?" A practice audit programme of this kind should examine accessibility, the process of care in a 